Localizador: 18047 doi: $10.35366 / 91763$

\title{
Esporotricosis cutánea linfangítica tratada con yoduro de potasio y criocirugía
}

\section{Lymphangitic cutaneous sporotrichosis in treatment with cryosurgery and potassium yodide}

José de Jesús Valdez-Velasco, ${ }^{*}$ Mirta de Lourdes Pérez-Olivos, Jessica Fabiola Valdez-Bernal, ${ }^{\S}$ Jorge Mayorga-Rodríguez

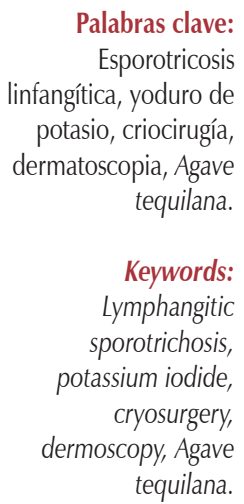

* Dermatólogo

Residente de Dermatología.

Médico Pasante.

Jefe del Centro de

Referencia de Micología (CEREMI).

Conflicto de intereses:

Ninguno.

Fuentes de financiamiento:

Autofinanciamiento.

Recibido:

06/Julio/2018

Aceptado:

08/Octubre/2018

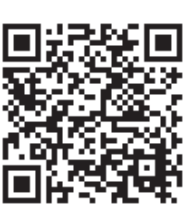

RESUMEN

La esporotricosis es una micosis subcutánea o profunda, producida por un complejo de hongos denominados Sporothrix schenckii. Afecta piel y tejido linfático. Se presenta un hombre de 34 años, campesino, quien muestra dermatosis que afecta brazo derecho, constituida por gomas y una placa verrugosa, de 10 semanas de evolución, posterior al traumatismo con espina de Agave tequilana. A la dermatoscopía se observó placa eritematosa, con costra melicérica café claro en su centro y halo eritemato-violáceo perilesional. Se aisló cepa del complejo Sporothrix schenckii, con diagnóstico de una esporotricosis linfangítica. Se manejó con yoduro de potasio y como coadyuvante criocirugía semanal con técnica spray, durante tres sesiones, con curación completa en nueve semanas. Resaltamos en este caso: la terapia combinada de yoduro de potasio con criocirugía, el uso de la dermatoscopia como herramienta auxilar de diagnóstico e inoculación traumática con espina de Agave tequilana, que no había sido descrita.

\section{ABSTRACT}

Sporotrichosis is a subcutaneous or deep mycosis, caused by a complex of fungi called Sporothrix schenckii, affecting skin and lymphatic tissue. It presents a male of 34 years, peasant. With dermatosis that affects the right arm, constituted by gums, and a warty plaque, of 10 weeks of evolution, after the trauma with the spine of Agave tequilana. A dermatoscopy revealed an erythematous plaque with a light brown melicric crust at its center and a perilesional erythematous-violaceous halo. It was isolated, strain of the Sporothrix schenckii complex, with diagnosis of a lymphangitic sporotrichosis. It was treated with potassium iodide and as a coadjuvant weekly cryosurgery with spray technique, during 3 sessions, with complete healing in 9 weeks. We highlight in this case: the combined therapy of potassium iodide with cryosurgery, the use of dermatoscopy as an auxiliary diagnostic tool and traumatic inoculation with Agave tequilana spine, which had not been described.

\section{INTRODUCCIÓN}

$\mathrm{L}$ a esporotricosis es una micosis subcutánea o profunda de curso subagudo o crónico, producida por un hongo dimórfico del complejo Sporothrix schenckii, afecta piel y tejido linfático en forma de nódulos y gomas; rara vez huesos, articulaciones y otros órganos. ${ }^{1-3}$ La infección se adquiere por traumatismo con material contaminado por el hongo e inusualmente por inhalación. ${ }^{1,4}$

Afecta a ambos géneros; el grupo etario más observado son los menores de 15 años (30\%), seguido por los adolescentes y adultos jóvenes de 16 a 45 años (32.61\%)..$^{1-3}$

El 93\% de los pacientes provienen de áreas rurales, aunque en los últimos años la enfermedad, al parecer, cambió su perfil y se observa con mayor frecuencia en zonas urbanas. La descomposición de materia vegetal en áreas de alta humedad con temperaturas entre 16 y $20{ }^{\circ} \mathrm{C}$, parecen ser las condiciones ideales para la proliferación de este hongo. Las astillas y espinas son hábitats preferidos y favorecen su crecimiento. Los trabajadores con mayor riesgo son: floristas, jardineros, trabajadores forestales, mineros y personas cuyas labores incluyen el manejo del suelo. . $^{3,5,6}$

La esporotricosis cutánea se presenta en tres variedades clínicas: a) linfangítica (75\%), se localiza en extremidades superiores (53\%), inferiores (18\%) y cara (21\%), esta última más común en niños (40 a 60\%) ; b) fija, se presenta como una placa verrugosa, infiltrada, en el lugar de inoculación y c) cutánea diseminada, forma rara de manifestación clínica, caracterizada por la presencia de tres o más lesiones que involucran dos o más áreas anatómicas. 1-3,7,8 


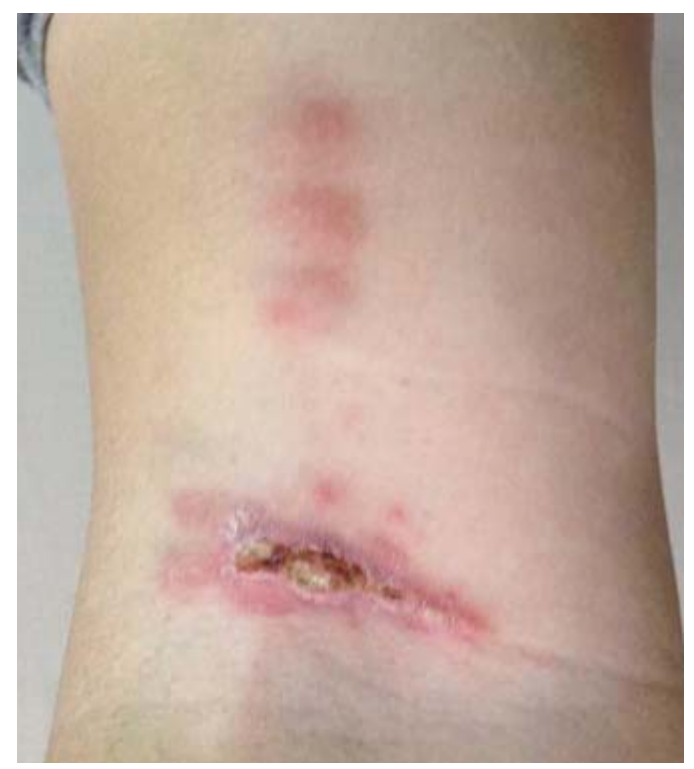

Figura 1: Dermatosis en brazo derecho, constituida por gomas, placas verrugosas, con superficie costrosa, siguiendo trayecto linfático.

Los azoles sistémicos son el tratamiento de elección para las formas cutáneas no complicadas (fija o linfangítica) y las osteoarticulares. Sin embargo, en los países de América Latina es ampliamente utilizado el yoduro de potasio (KI) para estas formas cutáneas, formulándolo a una concentración de $1 \mathrm{~g} / \mathrm{mL}$ y se administra a una dosis de 3 a $6 \mathrm{~g} /$ día, dividido en tres dosis por tres a cuatro meses aproximadamente, hasta la resolución de las lesiones y mantener de dos a cuatro semanas más; con este esquema se han reportado tasas de curación de 89 a 100\%, con un nivel de evidencia II-A., ${ }^{3,9-15}$

En caso de persistencia de las lesiones o cuando hay contraindicaciones, la criocirugía es una opción, ya que el hongo es sensible a temperaturas extremas. ${ }^{10}$ Un estudio sobre el tratamiento de la esporotricosis en humanos que utilizan nitrógeno líquido arrojó buenos resultados. .14,16,17

\section{PRESENTACIÓN DEL CASO}

Paciente masculino de 34 años, originario y residente de Tepatitlán, Jalisco, de ocupación campesino (jimador de agave), sin antecedentes de importancia.

Presenta dermatosis que afecta brazo derecho (cara interna a nivel de pliegue flexor), constituida por múltiples gomas de $3 \times 5 \mathrm{~cm}$ de diámetro, algunas de ellas forman una placa verrugosa, con superficie costrosa, las cuales siguen un trayecto ascendente y lineal (Figura 1), de 10 semanas de evolución, posterior al pinchazo con espina de agave (Agave tequilana). A la dermatoscopía se observó placa eritematosa, en su centro con costra melicérica café claro y un halo eritemato-violáceo perilesional (Figura 2).

De la secreción de las lesiones, se practicó cultivo en agar Sabouraud simple y modificado, se aisló cepa del complejo Sporothrix schenckii. El diagnóstico integral fue de una esporotricosis cutánea linfangítica. Se inició manejo con yoduro de potasio a una dosis de $6 \mathrm{~g} /$ día, dividido en tres dosis por dos meses y, como coadyuvante, el uso de criocirugía semanal con técnica spray, avanzando con

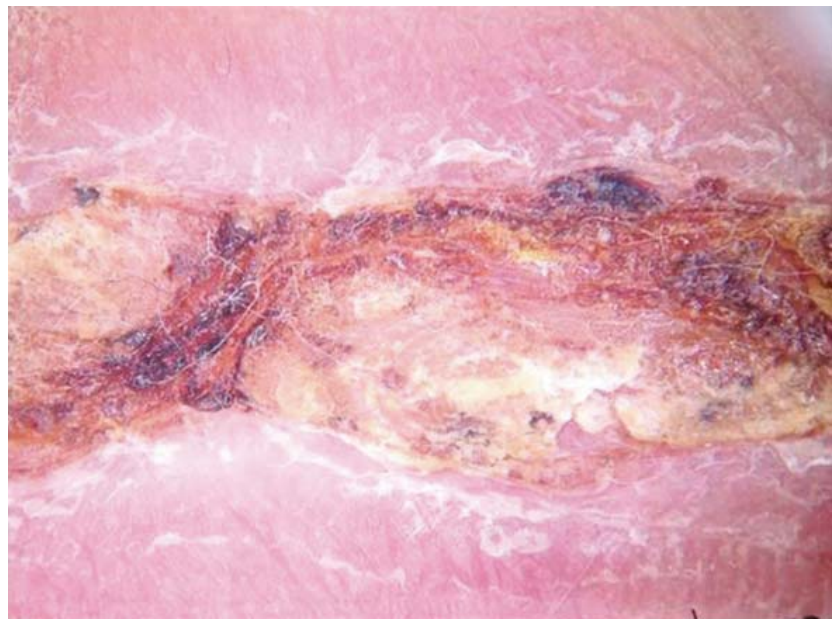

Figura 2: Dermatoscopía donde se observa placa eritematosa, costra melicérica café claro y halo eritemato-violáceo perilesional.

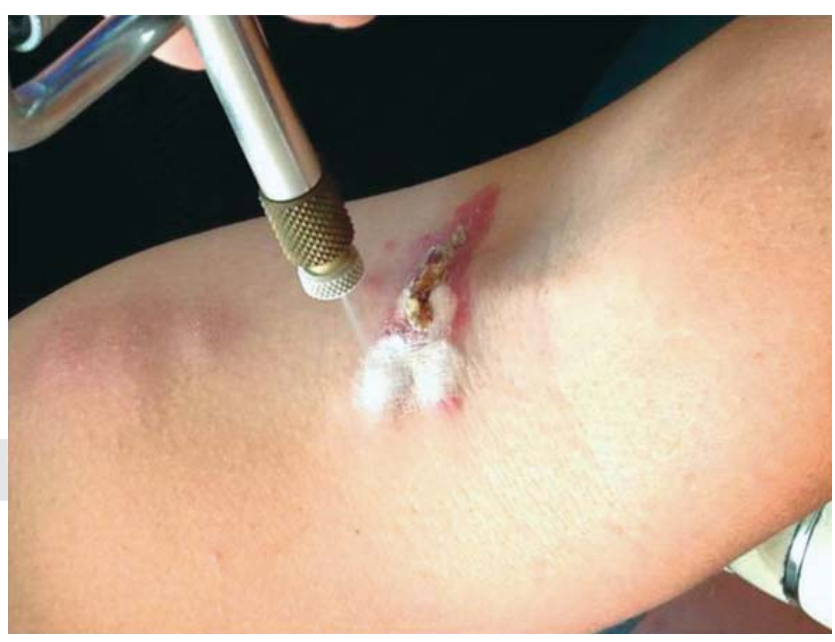

Figura 3: Criocirugía con técnica spray, dos ciclos de congelacióndescongelación de 1 minuto (tres sesiones). 


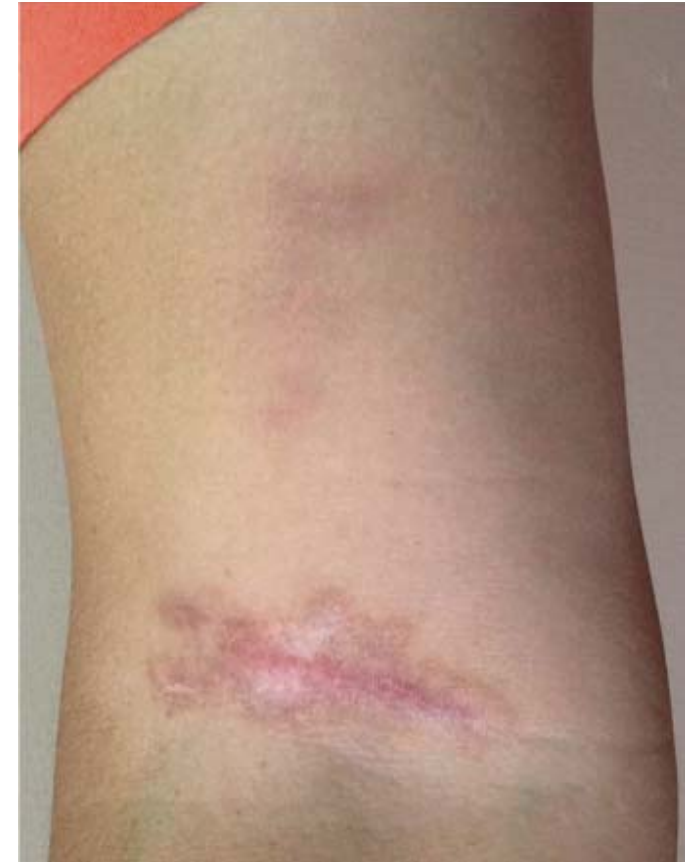

Figura 4: Postratamiento (nueve semanas), donde se observa sólo cicatriz atrófica.

el frente de congelación de toda la lesión, dos ciclos de congelación-descongelación de 1 minuto, con un total de tres sesiones (Figura 3). Obteniendo mejoría clínica completa en nueve semanas (Figura 4), con seguimiento dermatoscópico, en el cual se observa cicatriz residual (Figura 5), con cultivo y estudio directo negativo.

\section{DISCUSIÓN}

La criocirugía es una técnica coadyuvante en lesiones cutáneas de esporotricosis, junto a los antifúngicos sistémicos, cuyo propósito es optimizar el tiempo y duración del tratamiento, minimizando costos y evitando efectos adversos comunes. ${ }^{16-19}$ Además de ser un método terapéutico seguro en mujeres embarazadas, con una recomendación de evidencia B-III. ${ }^{18,20}$

Esta forma de tratamiento se basa en la destrucción selectiva de células o tejidos a través de un proceso de congelación, llegando de -20 a $-30{ }^{\circ} \mathrm{C}$, lo que resultará en una lesión necrótica. Los métodos de aplicación de la criocirugía dependen del tipo, forma, tamaño y ubicación de la lesión. Las técnicas que se pueden utilizar son: pulverización intermitente y controlada. El tiempo de congelación va de 3 a 60 segundos y varía según el tipo de lesión, formando un halo de congelación que oscila entre 3 y $4 \mathrm{~mm}$. En general, se recomienda un tiempo de 30 segundos para las lesiones planas y aplicarlo en tres ciclos de 10 segundos. Mientras que para las lesiones sobre-elevadas, un tiempo aproximado de 60 segundos, aplicado en seis ciclos de 10 segundos. Ambas con un intervalo de 4 minutos entre cada ciclo y reaplicando sesiones cada 30 días. ${ }^{16-20}$ En nuestro caso, puesto que el paciente presentaba una lesión elevada, cumplió el criterio para aplicar ciclos de 60 segundos de congelación y descongelación cada semana. ${ }^{21}$

Ferreira y colaboradores (2010) describen nueve casos con esporotricosis linfocutánea y localizada: un paciente tratado con yoduro de potasio, cinco con itraconazol y tres con terbinafina; además, terapia adyuvante de nitrógeno líquido. En cuanto al género, siete fueron mujeres y dos hombres, edad media de 45.8 años. Aplicación de una a cuatro sesiones mensuales de nitrógeno líquido, con dos ciclos de 15 segundos, con halo de congelación de $5 \mathrm{~mm}$. Todos los esquemas de tratamiento utilizados presentaron mejoría clínica. ${ }^{21}$

Bargma y su grupo (1995) describen tres casos de esporotricosis cutánea usando nitrógeno líquido. El primero fue tratado inicialmente con yoduro de potasio 10 gotas, tres veces al día, suspendido por intolerancia gástrica, aplicando criocirugía en 11 sesiones y crema tópica de ketoconazol al 2\%, con curación completa a los 24 meses. En el segundo solamente se aplicó nitrógeno líquido por 12 sesiones, durante un periodo de 29 semanas, con desaparición de la lesión. El tercer caso se trató sólo con nitrógeno líquido, cuatro sesiones, por ocho semanas, remitiendo la lesión. ${ }^{17}$

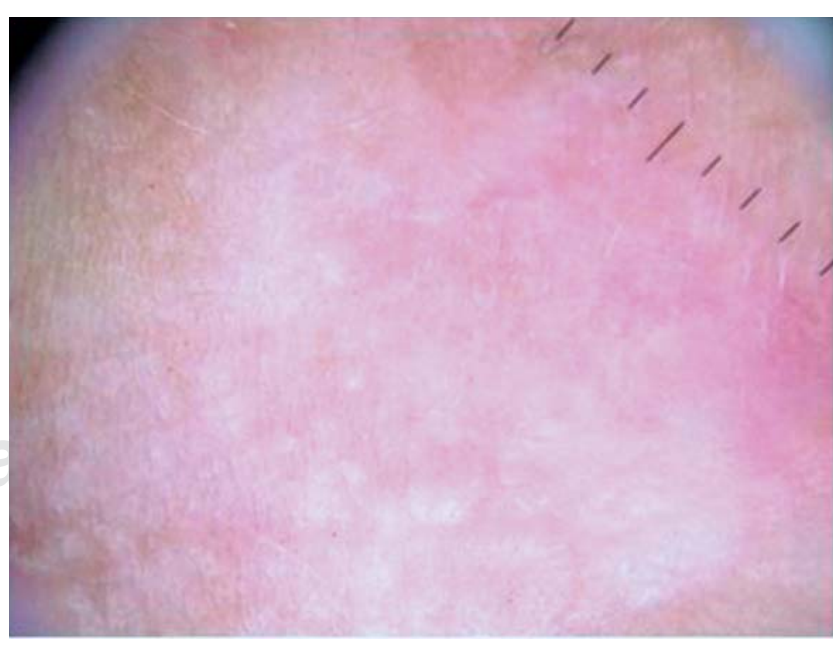

Figura 5: Seguimiento dermatoscópico (nueve semanas), se observa cicatriz residual. 
Fichman y colegas (2018) realizaron un estudio retrospectivo, el cual describe la respuesta clínica y el resultado de la criocirugía para el tratamiento de la esporotricosis cutánea en cuatro mujeres embarazadas. Todas las pacientes estaban en el segundo trimestre del embarazo y su edad varió de 18 a 34 años. Las sesiones se llevaron a cabo mensualmente y el número de éstas varió de una a tres. En cada sesión, las lesiones se trataron con dos ciclos de 10 a 30 segundos de tiempo de congelación con nitrógeno líquido en forma de spray. Todas las pacientes alcanzaron una curación clínica completa y no se observaron reacciones adversas durante el tratamiento ni recaídas. ${ }^{20}$

La importancia del presente caso radica, en primer lugar, en la sensibilidad de Sporothrix schenckii a las temperaturas bajas del nitrógeno líquido, que puede ser utilizado de forma sola o adyuvante con antimicóticos sistémicos, en ambas con buena respuesta clínica.,20-22
En segundo lugar, el uso de la dermatoscopia como auxiliar del diagnóstico, ya que en ésta infección de origen micótico ha sido poco utilizada.

$\mathrm{Y}$ en tercer lugar, describimos por primera vez la inoculación traumática de una espina de Agave tequilana, como fuente de contagio para el desarrollo de esporotricosis.

\section{CONCLUSIONES}

La criocirugía es una herramienta coadyuvante en pacientes con esporotricosis cutánea fija y linfangítica, como se demostró en este caso, combinada con el uso de yoduro de potasio.

\author{
Correspondencia: \\ Dra. Mirta de Lourdes Pérez Olivos \\ E-mail: mirprzo89@gmail.com
}

\section{BIBLIOGRAFÍA}

1. Bravo T. Esporotricosis: avances recientes en el diagnóstico de laboratorio, histopatología y la epidemiología en México. Rev Latinoamer Patol Clin. 2012; 59 (3): 147-171.

2. Alvarado CA, Arias CW. Esporotricosis. Revista CIEZT. 2016; 16 (1): 147-156.

3. Gaviria-Giraldo CM, Cardona-Castro N. Esporotricosis y cromoblastomicosis: revisión de la literatura. Rev CES Med. 2017; 31 (1): 77-91.

4. Bove-Sevilla PM, Mayorga-Rodríguez J, Hernández Hernández O. Esporotricosis transmitida por gato doméstico. Reporte de un caso. Med Cutan Iber Lat Am. 2008; 36: 33-35.

5. Bimbi C, Brzezinski P. Cutaneous sporotrichosis as an occupational disease: case report. Our Dermatol Online. 2017; 8 (1): 37-39.

6. Venadero-Albarrán F, Rhony-Orellana AA, Castillón-Alcaraz SJ, Bonifaz A, Padilla-Desgarennes MC. Esporotricosis linfangítica. A propósito de diferentes alternativas de tratamiento. Dermatol Rev Mex. 2010; 54 (3): 145-149.

7. Mayorga J, Martínez D, Méndez P. Aislamiento de Sporothrix schenckii en la naturaleza (suelos y plantas). Med Cutan Iber Lat Am. 1999; 27: 25-28.

8. Mayorga J, Barba Rubio J, Muñoz-Estrada VF, Rangel Corte?s A et al. Esporotricosis en el estado de Jalisco, estudio clínicoepidemiológico (1960-1966). Dermatol Rev Mex. 1997; 41: 105-108.

9. Arenas R. Micología médica ilustrada. $4^{\circ}$ ed. Ciudad de México: McGraw-Hill Interamericana; 2011. pp. 147-158.

10. Song Y, Li SS, Zhong SX, Liu YY, Yao L, Huo SS. Report of 457 sporotrichosis cases from Jilin province, northeast China, as serius endemic region. J Eur Acad Dermatol Venerol. 2013; 27: 313-318.

11. Mahajan VK. Sporotrichosis: an overview and therapeutic options. Dermatol Res Pract. 2014; 272376. doi:10.1155/2014/272376.

12. Bustamante B, Campos PE. Sporotrichosis treatment: overview and update. Curr Fungal Infect Rep. 2011; 5: 42-48.
13. Macedo PM, Lopes-Bezerra LM, Bernardes-Engemann AR, Orofino-Costa R. New posology of potassium iodide for the treatment of cutaneous sporotrichosis: study of efficacy and safety in 102 patients. J Eur Acad Dermatol Venereol. 2015; 29: 719-724.

14. Kauffman CA, Bustamante B, Chapman SW, Pappas PG. Clinical practice guideline for the management of sporotrichosis: 2007 update by the Infectious Disease Society of American, IDSA Guidel. Manag. Sporotrichosis. 2007; 45: 1255-1265.

15. Reis ÉG, Schubach TM, Pereira SA, Silva JN, Carvalho BW, Quintana MS, Gremião ID Association of itraconazole and potassium iodide in the treatment of feline sporotrichosis: a prospective study. Med Mycol. 2016; 54 (7): 684-690.

16. Souza CP, Lucas R, Ramadinha RH, Pires TB. Cryosurgery in association with itraconazole for the treatment of feline sporotrichosis. J Feline Med Surg. 2016; 18 (2): 137-143.

17. Bargman H. Successful treatment of cutaneous sporotrichosis with liquid nitrogen: report of three cases. Mycoses. 1995; 38 (7-8): 285-287.

18. Secchin P, Seabra GRP, Azulay DR, Fernandes NC, Ishida CE. Cryosurgery as an adjuvant treatment in sporotrichosis: a tree case report. Surg Cosmet Dermatol. 2017; 9 (3): 255-258.

19. Castillo R, et al. Guía de uso de la criocirugía en atención primaria. Med Fam. 2002; 2(3): 114-122.

20. Fichman V, Valle ACFd, de Macedo PM, Freitas DFS, Oliveira MMEd, Almeida-Paes $R$ et al. Cryosurgery for the treatment of cutaneous sporotrichosis in four pregnant women. PLoS Negl Trop Dis. 2018; 12 (4): e0006434.

21. Ferreira $\mathrm{CP}$, Galhardo MC, Valle AC. Cryosurgery as adjuvant therapy in cutaneous sporotrichosis. Braz J Infect Dis. 2011; 15 (2): 181-183.

22. Mackinnon JE, Conti-Diaz IA. The effect of temperature on sporotrichosis. Sabouraudia. 1962; 7: 38-45. 